\title{
The assessment of lens opacities in clinical practice: results of a national survey
}

\author{
N A Frost, J M Sparrow
}

\begin{abstract}
Aim-To investigate the examination of lens opacities in routine ophthalmic clinical practice.

Method-A questionnaire survey was mailed to 703 consultant ophthalmologists in the UK. The surgeons were asked which lens feature(s) they assessed in their clinics when deciding whether to offer cataract surgery.

Results-489 replies were received. A broad range of lens opacities was assessed, with differences between surgeons for some opacities with high prevalences in the population, particularly cortical opacities. Many (74\% of 467) surgeons assessed one or more lens opacities (anterior subcapsular cataract, vacuoles, water clefts, coronary flakes, focal dots, retrodots, fibre folds) which may be visually important but which have received relatively little attention by researchers.

Conclusions-Some classes of lens opacity which are traditionally measured by researchers may be ignored in clinical practice and opacities which are traditionally ignored by some researchers are regarded as clinically important by a substantial number of surgeons.

(Br F Ophthalmol 2001;85:319-321)
\end{abstract}

Department of

Ophthalmology,
University of Bristol,

UK

N A Frost

J M Sparrow

Correspondence to:

Mr NA Frost, Bristol Eye

Hospital, Lower Maudlin

Street, Bristol BS1 2LX, UK

Andy.Frost@bristol.ac.uk

Accepted for publication 24 October 2000

Examination of the human ocular lens is necessary to detect the presence of opacities and is essential to the diagnosis of cataract, but lens examination in clinical practice has received little attention by researchers. Little is known about the class or severity of cataracts listed for surgery. ${ }^{12} \mathrm{~A}$ variety of different opacities may be observed in the ageing lens ${ }^{34}$ and it is likely that some opacities have little or no effect on vision. The aim of this study was to provide information about which classes of

Table 1 Questions on lens morphology

Q1 When deciding whether to offer cataract surgery which lens feature(s) do you assess in your clinics? (tick one or more as appropriate)

Anterior subcapsular opacity Nuclear light scatter (opalescence)

Coronary flakes

Cortical spokes

Fibre folds

Focal dots

Posterior subcapsular opacity

Retrodots

Vacuoles

Waterclefts

Nuclear colour (brunescence) Any other feature(s): please name

Q2 When deciding whether to offer cataract surgery do you attach more importance to centrally located lens opacities (those closer to the visual axis) as opposed to peripherally located ones? (Yes/No)

Q3 Apart from posterior subcapsular cataract, do you consider the anteroposterior location of opacities when deciding whether to offer cataract surgery? (Yes/No)

Q4 When deciding whether to offer cataract surgery do you routinely grade cataracts in your clinics according to a recognised system-eg, Oxford, LOCS? (Yes/No) If yes: what is the name of the system? lens opacity are assessed by surgeons when deciding whether to offer cataract extraction.

\section{Methods}

A questionnaire survey was mailed to consultant ophthalmologists practising in the UK in 1997. The questionnaire comprised two sections. The first section inquired about lens morphology. The questions are listed in Table 1. The second section inquired about the use of vision tests and has been reported elsewhere. ${ }^{5}$ The surgeons were asked which lens feature(s) they assessed in their clinics when deciding whether to offer cataract surgery. A total of 703 questionnaires were mailed. An additional 38 local consultant ophthalmologists were excluded in advance from the survey. These surgeons had already completed pilot versions of the questionnaire.

\section{Results}

Of 703 questionnaires mailed 489 replies were received. Sixteen of the respondents excluded themselves because they did not consider the questionnaire relevant to their current practice. The commonest reason was that they did not routinely operate on the age group specified. A total of 473 of the respondents considered themselves eligible for inclusion in the study, of which 471 completed the section on lens morphology. Question 1, which concerned classes of lens opacity, was completed by 467 surgeons. The results from question 1 are presented in Table 2.

A broad range of lens opacities was assessed, with differences between surgeons for some opacities with high prevalences in the population, particularly cortical opacities. Because of the possibility that individual lens features were being disregarded on the questionnaire because other associated opacities had already been endorsed the responses were grouped into categories of "subcapsular", "nuclear sclerosis", and "cortical" (Table 3). Grouping the responses made little difference to the results

Table 2 Number of surgeons who indicated that they assessed a particular class of lens opacity when considering whether to offer cataract surgery

\begin{tabular}{lll}
\hline Class of opacity & $\begin{array}{l}\text { Number of } \\
\text { surgeons }\end{array}$ & $\%^{*}$ \\
\hline Posterior subcapsular opacity (PSC) & 451 & 97 \\
Nuclear colour, brunescence (NC) & 397 & 85 \\
Nuclear light scatter, opalescence (NO) & 323 & 69 \\
Cortical spokes (CSP) & 315 & 67 \\
Anterior subcapsular opacity (ASC) & 308 & 66 \\
Vacuoles & 165 & 35 \\
Water clefts (WC) & 133 & 28 \\
Coronary flakes & 95 & 20 \\
Focal dots & 82 & 18 \\
Retrodots & 68 & 15 \\
Fibre folds (FF) & 56 & 12
\end{tabular}

$\star$ Percentage of 467 respondents. 
Table 3 Number of surgeons who indicated that they assessed one or more of a specified group of opacities when considering whether to offer cataract surgery

\begin{tabular}{lll}
\hline Group of opacities * & $\begin{array}{l}\text { Number of } \\
\text { surgeons }\end{array}$ & $\%+$ \\
\hline Subcapsular opacities (ASC or PSC) & 452 & 97 \\
Nuclear sclerosis (NC or NO) & 433 & 93 \\
Cortical opacities (CSP, WC, FF) & 331 & 71
\end{tabular}

* See Table 2 for abbreviations.

+Percentage of 467 respondents.

for cortical opacities. Twenty nine per cent of surgeons did not endorse any of the questionnaire options for cortical spokes or other related opacities.

A total of $347(74 \%)$ of 467 surgeons indicated that they assessed one or more of the following lens opacities: anterior subcapsular cataract, vacuoles, water clefts, coronary flakes, focal dots, retrodots, fibre folds, which have been disregarded in many research studies. Other lens features were mentioned infrequently. Other features named were the zones of discontinuity (one surgeon), polychromatic lustre (one), lens shape-for example, lenticonus (two) and central nuclear morphology (one). Some surgeons also included features related to the ease of surgery-for example, lens stability.

The vast majority of surgeons (433 of 469) considered proximity to the visual axis to be an important feature. Opinion was divided as to the importance of the anteroposterior location of opacities (excluding posterior subcapsular cataract); 252 of 466 surgeons considered this feature. Ten of 466 routinely used a recognised grading system in their clinics. A further two used a recognised system occasionally and a further surgeon used part of the LOCS system.

\section{Discussion}

The highest endorsement frequency was for posterior subcapsular cataract $(97 \%)$. There is little doubt that posterior subcapsular cataract impairs vision. The class of opacity which is arguably the least likely to impair vision (fibre folds) was assessed by the smallest number of surgeons $(12 \%)$. The appearances of fibre folds may be caused by reflection ${ }^{6}$ and may not necessarily cause degradation of the retinal image. Between the above extremes there was a range of endorsement frequencies for the various lens features.

The questionnaire response to cortical spokes was interesting. Some investigators have reported associations between cortical opacity and visual impairment ${ }^{78}$ but this finding has not been consistent across all studies. ${ }^{910} \mathrm{~A}$ relatively low prevalence of "pure" cortical cataract was found in cases listed for surgery in Parma, Italy. ${ }^{1}$ Elliott et al found that some of their patients with extensive cortical opacity retained excellent visual acuity and attributed this to the ability to look through a "hole" in the cataract. ${ }^{11}$ Rouhiainen et al found that a decrease in Snellen acuity was associated with increasing nuclear opacification but not with early cortical opacity. ${ }^{10}$ Pesudovs and Coster found that a score of visual disability in cataract patients was related to nuclear opacity and to posterior subcapsular cataract but not to cortical cataract. ${ }^{12}$

It is also noteworthy that $74 \%$ of surgeons assessed lens opacities which have been disregarded in many research studies. It is commonly assumed that there are three major types of cataract-nuclear, cortical, and posterior subcapsular. ${ }^{13}$ This simplified scheme may be satisfactory for studies of severe cataract but it clearly does not take into account the subtleties of lens examination in clinical ophthalmic practice.

The results raise the question of which of the less frequently investigated classes of opacity cause visual impairment. If some, but not all, classes of opacity interfere with vision, careful examination of the lens at the slit lamp becomes crucial. Vision tests and visual symptoms may not be helpful in the presence of other coexisting ocular pathology. Discovery of lens opacities which are not visually significant would lead to a search for other causes of visual impairment. A possible means of avoiding the problem of classifying opacities is to assess the clarity of the clinician's view of the retina through the cataractous lens. Target resolution ophthalmoscopes have been devised for this purpose,${ }^{14}$ but these instruments provide an incomplete description of the severity of opacity. Nor do they discriminate between the optical effects of cataract and those of other structures within the eye. ${ }^{15}$ Likewise, it is uncertain whether assessment of the general clarity of the red reflex (for example, by direct ophthalmoscopy or retinoscopy) will reliably discriminate between visually significant early lens opacities and visually insignificant opacities. Some abnormalities are poorly visible on retroillumination.

The present survey has several implications for cataract research. Researchers frequently recruit cases of cataract from surgical waiting lists for a variety of purposes including psychophysical and epidemiological studies. Occasionally lenses are obtained after cataract extraction or from postmortem eyes for the purposes of biochemical or photometric analyses. The present results demonstrate the potential pitfalls of this approach if the required classes of opacity are not subsequently verified using standardised methods of lens examination. The presence of aphakia or pseudophakia may be included when estimating the prevalence of cataract in epidemiological studies. ${ }^{16}{ }^{17}$ This may lead to the anomalous situation where certain classes of opacity are discounted in phakic individuals but may be counted as cataract in those having received surgery. The event of cataract surgery has also be assumed to indicate progression of the opacity type measured in a longitudinal study. ${ }^{18}$ This assumption is not valid if coexisting but unmeasured classes of opacity have developed or progressed.

Although some classes of opacity are associated with one another ${ }^{19}$ it is presently uncertain whether the associations are strong enough to eliminate the potential measurement errors caused by ignoring particular opacities. Recently the Melton Eye Study has provided 
information about the prevalence of 11 clinicopathological classes of lens opacity. ${ }^{20}$ Such information, when combined with future knowledge of the vision impairing capabilities of a broader range of opacities, may help both in determining which lens features should be assessed and in evaluating the effects of ignoring specific classes of opacity.

We thank the UK Department of Health, NHS Executive South and West, the SAES study team, and all UK consultants who completed the questionnaire, or assisted with the pilot study.

1 Belpoliti M, Rosmini F, Carta A, et al. Distribution of cataract types in the Italian-American case-control study and at surgery in the Parma area. Ophthalmology 1995;102:15947 .

2 Klein BE, Klein R, Moss SE. Incident cataract surgery: the Beaver Dam Eye Study. Ophthalmology 1997;104:573-80.

3 Bron AJ, Brown NAP. Classification, grading and prevention of cataract. F Int Biomed Information Data 1983;4:2147.

4 Bron AJ, Brown NAP. Lens structure and forms of cataract. In: Duncan G, ed. The lens: transparency and cataract. EurIn: Duncan G,

5 Frost NA, Sparrow JM. Use of vision tests in clinical decision making about sests of a decision making about cataract surgery: results
national survey. Br f Ophthalmol 2000;84:432-4.

6 Brown NAP, Vrensen G, Shun-Shin GA, et al. Lamellar Brown NAP, Vrensen G, Shun-Shin GA, et al. Lamellar
separation in the lens: the case for fibre folds. Eye 1989;3: separation

7 Chylack LT, Padhye N, Khu P, et al. Loss of contrast sensitivity in diabetic patients with LOCS II classified cataracts. Br f Ophthalmol 1993;77:7-11.

8 Maraini G, Rosmini F, Graziosi P, et al. Influence of type and severity of pure forms of age-related cataract on visual acuity and contrast sensitivity. Invest Ophthalmol Vis Sci 1994;35:262-7.

9 Chylack LTJ, Jakubicz G, Rosner B, et al. Contrast sensitivity and visual acuity in patients with early cataracts. $\mathcal{F}$ Cataract Refract Surg 1993;19:399-404.

10 Rouhiainen P, Rouhiainen H, Salonen JT. The impact of early lens opacity progression on visual acuity and refraction. Ophthalmologica 1997;211:242-6.

11 Elliott DB, Hurst MA, Weatherill J. Comparing clinical tests of visual loss in cataract patients using a quantification of forward light scatter. Eye 1991;5:601-6.

12 Pesudovs K, Coster DJ. Cataract surgery reduces subjective visual disability. Aust NZ F Ophthalmol 1997;25(Suppl 1):S3-5.

13 Livingston PM, Carson CA, Taylor HR. The epidemiology of cataract: a review of the literature. Ophthalmic Epidemiol 1995;2:151-64.

14 Cotlier E, Fagadau W, Cicchetti DV. Methods for evaluation of medical therapy of senile and diabetic cataracts. Trans Ophthalmol Soc UK 1982;102:416-22.

15 Sharma YR, Vajpayee RB, Bhatnagar R, et al. A simple accurate method of cataract classification. Cataract-I. Ind accurate method of cataract classi

16 Hirvela H, Luukinen H, Laatikainen L. Prevalence and risk factors of lens opacities in the elderly in Finland: a population-based study. Ophthalmology 1995;102:108-17.

17 Leske MC, Connell AMS, Wu SY, et al. Prevalence of lens opacities in the Barbados Eye Study. Arch Ophthalmol 1997;115:105-11.

18 Leske MC, Chylack LTJ, Wu SY, et al. Incidence and progression of nuclear opacities in the longitudinal study of cataract. Ophthalmology 1996;103:705-12.

19 Thompson JR, Deane JS, Hall AB, et al. Associations between lens features assessed in the Oxford Clinical Cataract Classification and Grading System. Ophthalmic Epidemiol 1997;4:207-12.

20 Deane JS, Hall AB, Thompson JR, et al. Prevalence of lenticular abnormalities in a population-based study: Oxford clinical cataract grading in the Melton Eye Study. Ophthalmic Epidemiol 1997;4:195-206. 